\title{
DESEMPENHO DE MODELOS DE CALIBRAÇÃO DE GUIAS DE ONDA ACOPLADAS A TDR E A MULTIPLEXADORES EM TRÊS TIPOS DE SOLOS ${ }^{(1)}$
}

\author{
Eugenio Ferreira Coelho ${ }^{(2)}$, Lucas de Melo Vellame ${ }^{(3)}$, Mauricio Antonio \\ Coelho Filho $^{(2)} \&$ Carlos Alberto da Silva Ledo ${ }^{(2)}$
}

\begin{abstract}
RESUMO
O trabalho teve como objetivo avaliar modelos de calibração para dois tipos de guias de onda de TDR, referentes a dois equipamentos (Trase System e TDR 100), acopladas diretamente ao analisador de umidade ou a multiplexadores. Amostras de três tipos de solo foram acondicionadas em segmentos de tubos de PVC e saturadas. Dois tipos de guias de onda de três hastes, com capacitor e com resistor foram inseridas dentro de cada segmento de tubo com solo e conectadas a dois equipamentos de TDR, diretamente no testador de cabos ou via multiplexadores. Dados de umidade obtidos por gravimetria e da constante dielétrica foram tomados em cada coluna durante a secagem do solo da saturação até umidades próximas do limite inferior de disponibilidade de água por meio de leituras com as guias de onda conectadas ao testador de cabos e conectadas ao multiplexador. Um modelo polinomial cúbico foi ajustado aos dados da constante dielétrica do solo $(\varepsilon)$ e da correspondente umidade $(\theta)$ e cinco modelos de determinação de $\theta$ em função de $\varepsilon$ foram testados quanto ao desempenho. Os resultados mostraram que não houve diferença significativa na calibração das guias de onda com capacitor para uso com a TDR Trase System, considerando a conexão das guias ao analisador de umidade ou a multiplexadores. No caso da TDR 100, as guias de onda com resistor devem ser calibradas conforme o seu uso. O modelo cúbico foi o de melhor desempenho seguido pelo modelo de Roth que estimou, com boa exatidão, os valores da constante dielétrica e da umidade com $\alpha$ mais próximo de 0,5 para as guias de onda com capacitor que com as guias com resistor.
\end{abstract}

Termos de indexação: umidade do solo, constante dielétrica.

\footnotetext{
(1) Recebido para publicação em maio de 2004 e aprovado em dezembro 2005.

(2) Pesquisador da Embrapa Mandioca e Fruticultura. CEP 44380-000 Cruz das Almas (BA). E-mail: ecoelho@cnpmf.embrapa.br

${ }^{(3)}$ Estudante de Agronomia, Escola de Agronomia, Universidade Federal da Bahia - UFBA. CEP 44380-000 Cruz das Almas (BA). E-mail: lvellame@terra.com.br
} 


\title{
SUMMARY: PERFORMANCE OF CALIBRATION MODELS FOR TDR AND MULTIPLEXER - CONNECTED WAVEGUIDES IN THREE SOIL TYPES
}

\begin{abstract}
The study aimed at evaluating of calibration models for two kinds of TDR waveguides used with Trase System and TDR 100 equipments linked to the cable tester or the multiplexer. Disturbed samples of three soils were packed in PVC columns and, after soil saturation, two TDR waveguides of three rods with capacitor and with resistor were inserted into the soil columns. The waveguides were connected to two TDR equipments (Trase System and TDR 100), directly to the cable tester or a multiplexer. Soil water content data by gravimetry were collected during the drying process from saturation to values close to the lowest limit of water availability. The readings were performed with TDR probes connected to the cable tester and the multiplexer. A mathematical model was fitted to the bulk dielectric constant (e) and water content data $(q)$ and the performance of five models to obtain $q$ as a function of e was evaluated. Results showed that there was no significant difference between the TDR Trase System capacitor waveguide calibration connected either directly to the water content analyzer or the multiplexer channel. In case of TDR 100 using waveguides with resistor, the calibration has to be performed according to the connection of the waveguide to the water content analyzer or the multiplexer channel. The cubic calibration model performed best, followed by the Roth model, which estimated the values of bulk dielectric constant and water content well with a closer to 0.5 for waveguides with capacitor than for waveguides with resistor.
\end{abstract}

Index terms: soil water content, dielectric constant.

\section{INTRODUÇÃO}

O conhecimento do teor de água do solo é imprescindível a qualquer estudo que envolva as propriedades do sistema solo-água-planta. Sua determinação, pela técnica da reflectometria no domínio do tempo - TDR, é uma das mais exatas. O uso da TDR tem-se difundido na área de pesquisas principalmente por ser um método não-destrutivo, por sua exatidão e pela possibilidade de automação da coleta dos dados.

Todos os analisadores de umidade que funcionam pela TDR disponibilizam meios para se obter a velocidade de propagação do pulso eletromagnético (Vp) que, juntamente com a velocidade da luz (v), resulta na constante dielétrica aparente do solo $(\varepsilon)$ a partir da qual se determina a umidade do solo (Topp et al., 1980; Ledieu et al., 1986; Roth et al., 1990).

Os equipamentos de TDR podem funcionar com apenas uma guia de onda ou sonda a partir de leituras diretas no testador de cabos ou por meio de multiplexadores de leituras, que podem ser eletromecânicos ou eletrônicos. No caso da coleta contínua e automática dos dados em diversas posições no solo, há necessidade de uso de multiplexadores que requerem várias antenas conectadas ao testador de cabos. Tais equipamentos têm sido usados em diversos trabalhos referentes a manejo de solo e água (Baker \& Allmarras, 1990; Coelho \& Or, 1996).
As guias de onda, usadas para transmitir o pulso eletromagnético gerado no testador de cabos ao solo e daí determinar a constante dielétrica do solo, podem ser construídas de diferentes maneiras. Coelho et al. (2001) e Souza et al. (2001) indicam o uso de uma guia de onda de três hastes com um capacitor de $3,9 \mathrm{PiF}$ em paralelo com a malha externa (aterramento) e o fio interno (cabo de signal) para uso com a TDR Trase System. Vellame et al. (2003) indicam o uso de uma guia de onda similar à anterior com um resistor de $15 \Omega$ em série com o fio interno para uso com a TDR 100, construída de forma artesanal, o que requer avaliações.

$\mathrm{O}$ uso dos multiplexadores pode constituir um dos fatores de perturbação ao pulso eletromagnético, ou sinal emitido pela TDR, fato que se agrava, quando se utilizam sondas fabricadas em laboratórios. Em virtude do elevado custo das sondas de TDR industriais, as sondas têm sido produzidas em laboratórios a custos bem inferiores aos dos fabricantes, necessitando, porém, de calibração (Souza et al., 2001). O uso destas sondas em multiplexadores eletromecânicos e eletrônicos pode ter diferentes respostas do comportamento do sinal eletromagnético, na passagem por esses multiplexadores. Nas conexões nos multiplexadores, pode ocorrer aumento da impedância do sinal, provocando picos de reflexão que podem impedir as leituras automáticas feitas pelos aplicativos computacionais. O desenho de um multiplexador 
deve ser tal que os componentes de alta freqüência do sinal sejam atenuados ao máximo possível.

Não se tem conhecimento na literatura de trabalhos que avaliem o uso de sondas de TDR nãoindustriais acopladas a multiplexadores para determinação da umidade do solo pela TDR. Também não há referências na literatura sobre o efeito do tipo de equipamento de TDR com uso de sondas não-industriais na determinação da umidade do solo.

Os trabalhos relativos à calibração dos equipamentos de TDR têm mostrado inconsistência nos valores da constante dielétrica aparente $(\varepsilon)$, obtidos pela TDR em solos de texturas semelhantes ou diferentes (Tommaselli, 2001), em solos com altos teores de óxido de $\mathrm{Fe}$ e em solos de diferentes densidades e porosidades (Malicki et al., 1996). Os modelos de calibração da TDR podem ser de natureza empírica (Topp et al., 1980) e de natureza semi-empírica ou física, que envolvem modelos dielétricos mistos (Tommaselli, 2001; Noborio, 2001), em que $\varepsilon$ é fragmentada em seus componentes da matriz do solo, da água, que pode ser livre e adsorvida e do ar. Dentre esses modelos disponíveis, destacam-se os de Dobson et al. (1985), citados por Tommaselli (2001), e de Roth et al. (1990). Outros modelos envolvem parâmetros físicos do solo, tais como: os de Malicki et al. (1996) e Whalley (1993). O modelo de Topp et al. (1980) e o de Ledieu et al. (1986) têm sido muito usados, estando inseridos nos equipamentos de TDR dos fabricantes. Esses modelos são de caráter empírico e não levam em consideração os efeitos dos componentes da $\varepsilon$ e das propriedades físicas do solo, razão pela qual podem não ser os mais adequados.

A calibração desses equipamentos normalmente considera as guias de onda diretamente conectadas ao testador de cabos e não leva em conta a presença de multiplexadores. O uso de multiplexadores pode causar perdas no sinal e com isso afetar os valores da $\varepsilon$ do solo, principalmente quando forem usadas guias de onda não-industriais. O uso de multiplexadores tem sido reportado na literatura sem menção a calibrações. É necessário conhecer o comportamento das leituras da $\varepsilon$ com as guias de onda conectadas aos canais de multiplexadores, em comparação à conexão da guia de onda conectada diretamente à TDR.
O trabalho teve por objetivo avaliar, para três tipos de solo, modelos de calibração, usando dados experimentais obtidos com duas diferentes guias de onda de TDR, acopladas diretamente ou via multiplexadores a dois tipos de equipamentos de TDR.

\section{MATERIAL E MÉTODOS}

Três materiais de solo em amostras deformadas foram utilizados: S1-Latossolo Vermelho-Escuro eutrófico, de Tabuleiro Costeiro; S2- Neossolo fúlvico, solo aluvial da região norte de Minas Gerais; S3Latossolo Amarelo distrófico coeso (Quadro 1).

As amostras deformadas foram destorroadas e secas ao ar, sendo, em seguida, acondicionadas em colunas de PVC de $0,20 \mathrm{~m}$ de altura e $0,25 \mathrm{~m}$ de diâmetro. $\mathrm{O}$ solo foi acondicionado nas colunas de forma a resultar numa densidade média de $1,57 \mathrm{Mg} \mathrm{m}^{-3}$. O fundo ou a base de cada coluna de solo foi vedado com uma tela de malha fina e colocado para saturação durante $48 \mathrm{~h}$. Após atingir a saturação, a malha foi retirada e a extremidade tampada com um plástico para evitar perda de solo. Todo o material envolvido no processo, isto é, o solo seco ao ar, as colunas de PVC, os plásticos foram inicialmente pesados. Foram utilizadas três colunas de PVC com cada tipo de solo, num total de nove colunas. Em três colunas com mesmo tipo de solo (Figura 1), foram inseridas, após a saturação, duas guias de onda: uma, construída com um capacitor de 3,9 pF em paralelo com o fio interno e a malha de aterramento de cabo coaxial, e a outra, construída com um resistor de $15 \Omega$ em série com o terminal positivo do cabo coaxial, conforme especificações de Vellame et al. (2003) e Coelho et al. (2003).

Foram utilizados dois analisadores de umidade: um TDR Trase System (Soil Moisture), com acoplamento a um multiplexador de leituras de 16 canais, e um TDR 100 (Campbell Scientific Inc.) acoplado a dois níveis de multiplexadores (0 e 1) de oito canais por multiplexador. Uma vez inseridas as guias de onda no solo saturado, as colunas foram pesadas e postas a secar em condições naturais. As leituras de umidade e da constante dielétrica $(\varepsilon)$

\section{Quadro 1. Características texturais dos três tipos de solo utilizados na calibração das sondas de TDR}

\begin{tabular}{|c|c|c|c|}
\hline Tipo de solo & Areia total & Silte & Argila \\
\hline & 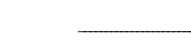 & $-\mathrm{g} \mathrm{kg}^{-1}$ & - \\
\hline S1 - Latossolo Vermelho-Escuro eutrófico & 480 & 320 & 200 \\
\hline $\mathrm{S} 2$ - Neossolo fúlvico & 550 & 310 & 140 \\
\hline S3 - Latossolo Amarelo distrófico coeso & 444 & 131 & 425 \\
\hline
\end{tabular}




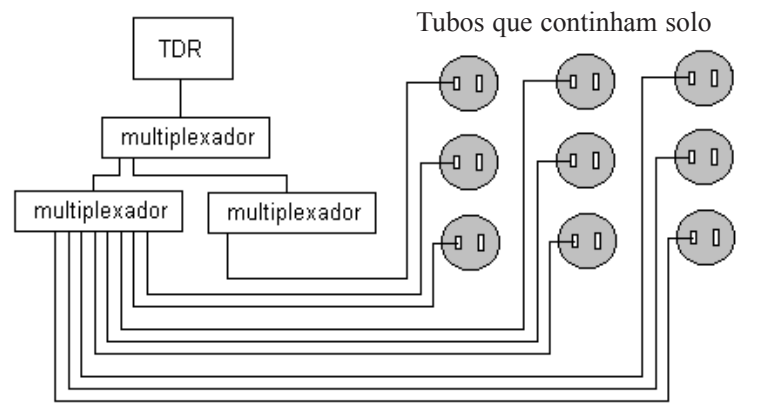

Figura 1. Disposição das guias de onda e do equipamento de TDR, com os multiplexadores.

foram feitas numa freqüência diária, seguidas de pesagens das colunas. As determinações das $\varepsilon$ foram efetuadas com as guias de onda acopladas diretamente ao testador de cabos ou ao multiplexador (Figura 1). As leituras foram feitas num computador por meio do aplicativo "PCTDR", no caso da TDR 100, e diretamente do equipamento, no caso da TDR Trase System. As leituras foram feitas até a umidade atingir valores próximos do limite inferior da disponibilidade de água do solo, quando as colunas foram levadas à estufa a $108{ }^{\circ} \mathrm{C}$ até atingir peso constante (PS). As umidades volumétricas tomadas a cada leitura $\left(\theta_{\mathrm{i}}\right)$ foram obtidas por gravimetria, isto é

$$
\theta_{\mathrm{i}}=\frac{P U_{\mathrm{i}}-\mathrm{PS}}{\mathrm{PS}-\mathrm{PE}} \cdot \frac{\mathrm{d}_{\mathrm{s}}}{\mathrm{d}_{\mathrm{a}}}
$$

em que $\mathrm{PU}_{\mathrm{i}}$ é o peso do solo úmido atual $+\mathrm{PE}$, sendo $\mathrm{PE}=$ peso da coluna de PVC + Peso das guias de onda + envoltórios (tela e plástico); PS é o peso do solo seco $+\mathrm{PE} ; \mathrm{d}_{\mathrm{b}}$ é a densidade do solo e $\mathrm{d}_{\mathrm{a}}$ é a densidade da água.

As estimativas de $\varepsilon$ em função de $\theta$ obtidas por cinco modelos de calibração foram comparadas aos dados de $\varepsilon$ lidas nos equipamentos de TDR e das umidades correspondentes em base volumétrica. O modelo de Roth et al. (1990) considera $\varepsilon$ como uma resultante de componentes da matriz do solo $\left(\varepsilon_{\mathrm{S}}\right)$, do ar $\left(\varepsilon_{\mathrm{a}}\right)$ e da água $\left(\varepsilon_{\mathrm{w}}\right)$ :

$$
\varepsilon^{\alpha}=\theta \varepsilon_{\mathrm{w}}{ }^{\alpha}+(1-\mathrm{P}) \varepsilon_{\mathrm{s}}^{\alpha}+(\mathrm{P}-\theta) \varepsilon_{\mathrm{a}}^{\alpha}
$$

em que $\mathrm{P}$ corresponde à porosidade total e $\theta$ à umidade do solo; $\alpha$ leva em conta os efeitos do arranjo geométrico dos componentes do meio na $\varepsilon$. O valor de $\alpha$ foi obtido por otimização do modelo, isto é, minimizando a função objetiva dada pela soma dos quadrados dos desvios entre os valores de $\theta$ estimados e medidos. O valor inicial de $\alpha$ foi de 0,5 conforme alguns autores (Topp et al., 1980; Ledieu et al., 1986; Roth et al., 1990; Malicki et al., 1996). Os valores de $\varepsilon_{\mathrm{S}}, \varepsilon_{\mathrm{a}}$ e de $\varepsilon_{\mathrm{w}}$, adotados em todos os cálculos, foram 5, 1 e 77, respectivamente. Malicki et al. (1996) propuseram um modelo relacionando a umidade e $\varepsilon$ com inclusão da densidade do solo, do tipo:

$$
\theta=\frac{\varepsilon^{0,5}-0,819-0,168 d_{S}-0,159 d_{S}^{2}}{7,17+1,18 d_{S}}
$$

O modelo de Topp et al. (1980) consiste numa polinomial do terceiro grau para estimativa dos teores de água, como função da $\varepsilon$ :

$\theta=-5,3 \times 10^{-2}+2,92 \times 10^{-2} \mathrm{e}-5,5 \times 10^{-4} \mathrm{e}^{2}+4,3 \times 10^{-6} \mathrm{e}^{3}(4)$

Além do modelo cúbico de Topp et al.(1980), foi proposto, neste trabalho, um modelo cúbico do tipo $\theta=\mathrm{a}+\mathrm{b} \varepsilon+c \varepsilon^{2}+\mathrm{d} \varepsilon^{3}$, em que os parâmetros foram determinados por otimização, e a soma dos quadrados dos desvios entre os valores observados e estimados de $\theta$ foi a função-objetiva. Whalley (1993) propôs um modelo do tipo:

$$
\sqrt{\varepsilon}=\theta\left(\sqrt{\varepsilon_{\mathrm{w}}-1}\right)+\frac{\mathrm{d}_{\mathrm{s}}}{\mathrm{d}_{\mathrm{p}}}\left(\sqrt{\varepsilon_{\mathrm{s}}-1}\right)+1
$$

em que se levou em consideração a densidade do solo e a densidade de partículas $\left(\mathrm{d}_{\mathrm{S}}\right)$, além de considerar os componentes da constante dielétrica aparente do solo $\left(\varepsilon_{\mathrm{S}}\right)$ e da água $\left(\varepsilon_{\mathrm{w}}\right)$, tendo sido os valores os mesmos usados na equação 2 . O modelo de Ledieu et al. (1986) é dado pela equação:

$$
\theta=0,1138 \sqrt{\varepsilon}-0,1758
$$

Os valores medidos e estimados pelos diferentes modelos de $\varepsilon$ para as duas guias de onda, para as leituras feitas com as guias de onda conectadas ao testador de cabos e ao multiplexador para os dois equipamentos de TDR (Trase System e TDR 100), foram comparados usando um desvio percentual médio dos desvios entre os valores medidos e estimados de cada leitura e pesagem correspondente (DV).

$$
\mathrm{DV}=\sum_{\mathrm{i}=1}^{\mathrm{n}} \frac{1}{\mathrm{n}}\left|\frac{\varepsilon_{\mathrm{oi}}-\varepsilon_{\mathrm{ei}}}{\varepsilon_{\mathrm{oi}}}\right| \cdot 100
$$

em que $\varepsilon_{\mathrm{oi}}$ e $\varepsilon_{\mathrm{ei}}$ são valores de $\varepsilon$ medidos e estimados; $\mathrm{n}$ é o número de leituras. Foi feita a análise de variância do experimento considerado fatorial 3 (tipos de solo) x 2 (tipos de guias de onda) x 2 (leitura com uso do testador de cabos e multiplexador) x 6 (modelos de calibração) com três repetições (três colunas de PVC para cada tipo de solo), considerando variáveis dependentes $\varepsilon$, o coeficiente de determinação $\mathrm{R}^{2}$ de ajuste dos modelos e a média dos desvios médios entre os valores reais e estimados de $\varepsilon$ como função de $\theta$ (DV).

\section{RESULTADOS E DISCUSSÃO}

A análise de variância mostrou rejeição da hipótese de nulidade para a variável dependente $\varepsilon$ 
com relação à fonte de variação tipo de solo, em que as constantes dielétricas dos três solos foram diferentes entre si a $5 \%$ pelo teste de Tukey (Quadro 2). O Latossolo Amarelo, de maior teor de argila e menor teor de silte, apresentou maior $\varepsilon$ que os outros solos, que estiveram mais próximos entre si. Este resultado reforça os resultados obtidos por alguns autores (Roth et al., 1990; Tommaselli, 1997), para os quais solos de textura fina influenciam a relação entre $\varepsilon$ e o teor de água do solo. Os resultados não condizem com aqueles obtidos por Ponizovsky et al. (1999) que observaram redução na $\varepsilon$ com o aumento do teor de argila. No caso da variável dependente coeficiente de determinação, a análise de variância foi significativa para tipo de solo, tipo de guia de onda, modelos de estimativa de $\varepsilon$, interação tipo de solo $\mathrm{x}$ modelo de estimativa, interação tipo de guia de onda x leitura com uso do testador de cabos e multiplexador.

Em geral, as médias dos coeficientes de determinação de todos os modelos testados para os três tipos de solo diferiram significativamente entre si $(\mathrm{P}<0,05)$, sendo o máximo valor referente ao

Quadro 2. Valores médios de $\varepsilon$ (constante dielétrica do solo) medidos para os três tipos de solo

\begin{tabular}{ll}
\hline \multicolumn{1}{c}{ Tipo de solo } & Média de $\varepsilon$ \\
\hline Latossolo Vermelho-Escuro eutrófico & $14,67 \mathrm{a}^{(1)}$ \\
Neossolo fúlvico & $17,76 \mathrm{~b}$ \\
Latossolo Amarelo coeso & $18,17 \mathrm{c}$ \\
\hline
\end{tabular}

(1) Médias de tratamentos seguidas por letras distintas, na coluna, diferem pelo teste de TuKey $(\mathrm{P}<0,05)$.
Latossolo Amarelo e o mínimo referente ao Latossolo Vermelho-Escuro (Quadro 3). O valor de DV foi menor para o Latossolo Amarelo que diferiu das médias para os outros tipos de solo $(\mathrm{P}<0,05)$ que, por sua vez, não diferiram entre si. Segundo tais resultados, tanto os modelos de natureza física como empírica tiveram melhor desempenho para um tipo de solo de textura mais fina, não concordando com os de Tommaselli (2001) que obteve resultados usando diferentes tipos de solo e que o levaram a concluir que os modelos físicos apresentam melhor ajuste para solos de textura grossa.

Verificou-se diferença significativa entre os coeficientes de determinação $(\mathrm{P}<0,05)$ referentes aos ajustes dos modelos aos dados gerados com uso dos dois tipos de guias de onda: a guia de onda com capacitor de uso na TDR Trase System resultou em maior coeficiente de determinação e menor desvio médio, comparada ao coeficiente de determinação e desvio médio da guia de onda com resistor construída para uso com a TDR 100 (Quadro 4). Isso indica uma calibração mais exata dos modelos para as guias com capacitores conectadas a TDR Trase System.

O modelo cúbico apresentou maior coeficiente de determinação e menores DV de $\varepsilon$, medidos pela TDR e estimados pelos modelos, seguido pelo modelo de Roth et al. (1990), do qual diferiu significativamente tanto pelos $\mathrm{R}^{2}$ como pelas médias dos DV (Quadro 5). Os coeficientes de determinação médios dos dois modelos diferiram dos demais, tendo o modelo de Malicki et al. (1996) resultado no terceiro maior coeficiente de determinação médio, diferindo significativamente dos modelos de Whalley (1993), Ledieu et al. (1986) e Topp et al. (1980) que não diferiram entre si para os DV (Quadro 5). Os valores de DV resultantes dos modelos de Whalley (1993) e

Quadro 3. Valores médios dos coeficientes de determinação de todos os modelos e dos DV (média dos desvios médios entre os valores reais e estimados de $\varepsilon$ como função da umidade do solo - $\theta$ )

\begin{tabular}{llc}
\hline \multicolumn{1}{c}{ Tipo de solo } & Média de $\mathbf{R}^{2}$ & DV (\%) \\
\hline Latossolo Vermelho-Escuro eutrófico & $0,6574 \mathrm{a}^{(1)}$ & $12,5 \mathrm{~b}$ \\
Néossolo fúlvico & $0,7310 \mathrm{~b}$ & $12,4 \mathrm{~b}$ \\
Latossolo Amarelo coeso & $0,8020 \mathrm{c}$ & 8,7 a \\
\hline
\end{tabular}

(1) Médias de tratamentos seguidas por letras distintas, na coluna, diferem pelo teste de TuKey $(\mathrm{P}<0,05)$.

Quadro 4. Valores médios dos coeficientes de determinação de todos os modelos e dos DV de $\varepsilon$ para os dois tipos de guia de onda

\begin{tabular}{ccc}
\hline Tipo de guia de onda & Média de $\mathbf{R}^{2}$ & Média do DV (\%) \\
\hline Com capacitor (Trase System) & $0,7752 \mathrm{a}$ & $11,1 \mathrm{a}$ \\
Com resistor (TDR 100) & $0,6395 \mathrm{~b}$ & $12,2 \mathrm{~b}$ \\
\hline
\end{tabular}

\footnotetext{
(1) Médias de tratamentos seguidas por letras distintas, na coluna, diferem pelo teste de TuKey $(\mathrm{P}<0,05)$.
} 
Ledieu et al. (1986) não diferiram entre si, tendo sido inferiores aos de Topp et al. (1980). Os valores de DV destes três modelos foram superiores aos dos modelos cúbicos, de Roth et al. (1990) e de Malick et al. (1996).

A análise do desdobramento da fonte de variação tipo de guia de onda dentro de cada nível de leitura (guia de onda conectada direto ao TDR e ao multiplexador) mostrou que as guias de onda com capacitor permitiram melhor ajuste dos dados pelos modelos do que as guias de onda com resistor (Quadro 6). Nesse caso, para a guia de onda conectada ao testador de cabos, não houve diferença significativa entre os desvios médios de $\varepsilon$, mas, para a conexão ao multiplexador, o DV foi menor para a guia de onda com capacitor $(\mathrm{P}<0,05)$. Fixando o tipo de guia de onda, no caso das guias com resistor, houve diferença significativa dos $\mathrm{R}^{2}$ e dos DV obtidos com as guias conectadas ao testador de cabos em relação aos obtidos com as guias conectadas ao multiplexador, com maiores valores do $\mathrm{R}^{2}$ e menores DV no primeiro caso. Para as guias de onda com capacitor, não houve diferença significativa nos $R^{2}$ e nos DV referentes às estimativas de $\varepsilon$ em função de $\theta$ para leituras feitas com as guias de onda conectadas ao testador de cabos e conectadas ao multiplexador. Assim, no caso da TDR Trase System com as guias de onda com capacitor, a calibração das guias de onda podem ser feitas tanto com as guias conectadas ao multiplexador como ao testador de cabos, enquanto as guias com resistor devem ser calibradas conforme a conexão das guias nos trabalhos a serem desenvolvidos.

O modelo cúbico com os coeficientes fixos, como é o caso do de Topp et al. (1980) e o de Ledieu et al. (1986), apresentou o mais baixo desempenho em geral para os três tipos de solo, sob quaisquer circunstâncias de uso avaliadas. A natureza empírica, que não leva em consideração as propriedades físicas e os componentes dielétricos do solo, considerando os coeficientes fixos, justifica o comportamento desses modelos. O modelo de Roth et al. (1990), de caráter mais físico (Or \& Wraith, 1997) e considerado semi-empírico (Tommaselli, 2001), apresentou um desempenho razoável, uma vez que seus parâmetros, incluindo o $\alpha$, foram fixados para todas as situações. O valor de $\alpha$ foi de 0,75067 para todos os materiais de solo, considerando a guia de onda com resistor (TDR 100). No caso da guia de onda com capacitor (Trase

Quadro 5. Valores médios dos $\mathrm{R}^{2}$ e DV resultantes da aplicação dos modelos para os três tipos de solo, dois tipos de guias de onda e leituras com as guias conectadas ao testador de cabos e ao multiplexador

\begin{tabular}{lcc}
\hline \multicolumn{1}{c}{ Modelo } & Média de $\mathbf{R}^{2}$ & Média do DV (\%) \\
\hline Topp et al. (1980) & $0,5221 \mathrm{e}^{1}$ & $16,7 \mathrm{~d}$ \\
Ledieu et al. (1986) & $0,5778 \mathrm{~d}$ & $15,2 \mathrm{~d}$ \\
Whalley (1993) & $0,5867 \mathrm{~d}$ & $7,9 \mathrm{~b}$ \\
Roth et al. (1990) & $0,8673 \mathrm{~b}$ & $12,4 \mathrm{c}$ \\
Malicki et al. (1996) & $0,7097 \mathrm{c}$ & 2,5 a \\
Cúbico & $0,9808 \mathrm{a}$ & \\
\hline (1) Médias de tratamentos seguidas por letras distintas, na coluna, diferem pelo teste de Scott $-\mathrm{Knot}(1974), \mathrm{P}<0,05$.
\end{tabular}

Quadro 6. Valores médios dos coeficientes de determinação e das médias dos DV, considerando a conexão da guia de onda e a variação do tipo de guia de onda

\begin{tabular}{llll}
\hline Conexão da guia de onda & Tipo de guia de onda & Média de R & Média do DV (\%) \\
\hline Testador de cabos & Com capacitor & $0,7817 \mathrm{a}$ & 10,6 a \\
Testador de cabos & Com resistor & $0,7002 \mathrm{~b}$ & $11,2 \mathrm{a}$ \\
Multiplexador & Com capacitor & $0,8177 \mathrm{a}$ & $10,2 \mathrm{a}$ \\
Multiplexador & Com resistor & $0,6209 \mathrm{~b}$ & $12,8 \mathrm{~b}$ \\
Testador de cabos & Com resistor & $0,7002 \mathrm{a}$ & $11,2 \mathrm{a}$ \\
Multiplexador & Com resistor & $0,6209 \mathrm{~b}$ & $12,8 \mathrm{~b}$ \\
Multiplexador & Com capacitor & $0,8177 \mathrm{a}$ & $10,2 \mathrm{a}$ \\
Testador de cabos & Com capacitor & $0,7817 \mathrm{a}$ \\
\hline
\end{tabular}

(1) Médias de tratamentos seguidas por letras distintas, na coluna, diferem pelo teste de TuKey $(\mathrm{P}<0,05)$. 
System), $\alpha$ foi igual a 0,5459 , para os Latossolos Amarelos e Latossolos Vermelho-Escuros, e igual a 0,4368 , para o solo Aluvial. Os valores de $\alpha$ para as guias de onda com capacitor estiveram mais próximos do valor de 0,50 mais comumente usado (Topp et al., 1980; Ledieu et al.,1986; Roth et al., 1990; Malicki et al., 1996) que o valor referente às guias de onda com resistor. O melhor desempenho do modelo cúbico em relação aos demais deve-se à flexibilidade dos parâmetros do modelo, que variaram com as características do solo, tipo de guia de onda e conexão da guia de onda, o que resultou em uma equação para cada situação.

Os resultados mostraram, portanto, que o uso de modelos pré-instalados, com coeficientes fixos, pode estar sujeito a imprecisões nas estimativas da umidade ou da $\varepsilon$ e que o tipo de guia de onda e sua conexão direta ao testador de cabos ou a multiplexadores exerce influência sobre o desempenho desses modelos.

\section{CONCLUSÕES}

1. O ajuste dos modelos de calibração de guias de onda de TDR aos dados de umidade volumétrica e da constante dielétrica do solo $(\varepsilon)$ variou conforme o tipo de solo, o tipo de guia de onda e a conexão da guia de onda ao testador de cabos.

2. O modelo cúbico pode ser calibrado com exatidão para cada situação, em termos de tipos de solo, de guias de onda e uso dessas guias direto no testador de cabos via multiplexadores; entretanto, a fixação dos coeficientes do modelo, como é caso do modelo de Topp et al. (1980), pode não resultar em calibrações precisas.

3. O modelo de Roth et al. (1990) ajustou com boa exatidão os dados de $\varepsilon$ e da umidade com a mais próximo de 0,5 para as guias de onda com capacitor.

4. O modelo de Ledieu et al. (1986) e o de Topp et al. (1980) foram os de mais baixo desempenho na estimativa da $\varepsilon$ em função da umidade.

5. No caso da TDR Trase System, para as guias de onda com capacitor, a calibração das guias de onda pode ser feita com a conexão das guias ao multiplexador ou ao testador de cabos.

6. No caso da TDR 100, para as guias com resistor, a calibração vai depender da conexão das guias ao testador de cabos ou ao multiplexador.

\section{LITERATURA CITADA}

BAKER, J.M. \& ALLMARAS, R.R. System for automating and multiplexing soil moisture measurement by Time Domain Reflectometry. Soil Sci. Soc. Am. J., 54:1-6, 1990.
COELHO, E.F.; CALDAS, R.C.; SANTOS, D.B. \& LEDO, C.A.S. Número e espaçamento entre hastes de guias de onda para medida de umidade do solo com TDR. R. Bras. Eng. Agric. Amb., 7:215-220, 2003.

COELHO, E.F.; ANDRADE, C.L.T.; OR, D.; LOPES, L.C. \& SOUZA, C.F. Desempenho de diferentes guias de ondas para uso com o analisador de umidade Trase. R. Bras. Eng. Agric. Amb., 5:81-87, 2001.

COELHO, E.F. \& OR, D. A parametric model for two-dimensional water uptake by corn roots under drip irrigation. Soil Sci. Soc. Am. J., 60:1039-1049, 1996.

DOBSON, M.C.; ULABY, F.T.; HALLIKAINEN, M.T. \& ELRAYES, M.A. Microwave dielectric behavior of wet soil: II Dielectric mixing models. IEEE Trans. Geo. Rem. Sens., 23:35-46, 1985.

LEDIEU, J.; DE RIDDER, P.; DE CLERCK, P. \& DAUTREBANDE, S. A method for measuring soil water moisture by time-domain reflectometry. J. Hydrol., 88:319$328,1986$.

MALICKI, M.A.; PLAGGE, R. \& ROTH, C.H. Improving the calibration of dielectric TDR soil moisture determination taking into account the solid soil. Eur. J. Soil. Sci., 47:357366,1996 .

NOBORIO, K. Measurement of soil water content and electrical conductivity by time domain reflectometry: A review. Comp. Electr. Agric., 31:213-237, 2001.

OR, D. \& WRAITH, J.M. Agricultural and enviromental soil physics. Logan, Utah State University, 1997. 218p.

PONIZOVSKY, A.A.; CHUDINOVA, S.M. \& PACHEPSKY, Y.A. Performance of TDR calibration models as affected by soil moisture. J. Hydrol., 18:35-43, 1999.

ROTH, C.H.; SCHULIN, R.; FLUHLER, H. \& ATTINGER, W. Calibration of time-domain reflectometry for water content measurement using composite dielectric approach. Water Res. Res., 26:2267-2273, 1990.

SCOTT, A.J. \& KNOTT, M.A. A cluster analysis method for grouping means in the analysis of variance. Biometrics, 30:507-512, 1974.

SOUZA, C.F.; MATSURA, E.E. \& TESTEZLAF, R. Experiência de laboratório de hidráulica, irrigação e drenagem da Faculdade de Engenharia Agrícola/UNICAMP no uso da técnica da TDR. In: MATSURA, E.E.; JAVAREZ JUNIOR,A.; GOMES, E.P. \& SOUZA, C.F., eds. Aplicações técnicas de TDR na agricultura. Campinas, FEAGRI/ UNICAMPI, 2001. p.147-176.

TOMMASELLI, J.T.G. Critérios para calibração de sondas de TDR. In: MATSURA, E.E.; JAVAREZ JUNIOR, A.; GOMES, E.P. \& SOUZA, C.F., eds. Aplicações técnicas de TDR na agricultura. Campinas, FEAGRI/UNICAMPI, 2001. p. $85-114$. 
TOMMASELLI, J.T.G. Influência de algumas características do solo sobre a calibração de um aparelho de TDR (Tyme Domain Reflectometry). Piracicaba, Escola Superior de “Agricultura Luiz de Queiroz", 1997. 109p. (Tese de Doutorado)

TOPP,G.C.; DAVIS, J.L. \& ANNAN, A. Electromagnetic determination of soil water content: Measurement in coaxial transmission lines. Water Res. Res, 16:576-583, 1980.
VELLAME, L.M.; COELHO, E.F.; COELHO FILHO, M.A. \& SANTOS, M.R. Guia de onda para estimativa da umidade do solo pela TDR com uso de multiplexadores In. CONGRESSO BRASILEIRO DE ENGENHARIA AGRÍCOLA, 32., Goiânia, 2003. Anais. Goiânia, Sociedade Brasileira de Engenharia Agrícola, 2003. CD-ROM

WHALLEY, W.R. Considerations on the use of time domain reflectometry (TDR) for measuring soil water content. J. Soil Sci., 44:1-9, 1993. 